\title{
Stochastic theory for the glassy state. 1. Influence of formation history on the glass transition temperature and equation of state
}

Citation for published version (APA):

Vleeshouwers, S. M., \& Nies, E. (1992). Stochastic theory for the glassy state. 1. Influence of formation history on the glass transition temperature and equation of state. Macromolecules, 25(25), 6921-6928.

https://doi.org/10.1021/ma00051a031

DOI:

10.1021/ma00051a031

Document status and date:

Published: 01/01/1992

Document Version:

Publisher's PDF, also known as Version of Record (includes final page, issue and volume numbers)

\section{Please check the document version of this publication:}

- A submitted manuscript is the version of the article upon submission and before peer-review. There can be important differences between the submitted version and the official published version of record. People interested in the research are advised to contact the author for the final version of the publication, or visit the $\mathrm{DOI}$ to the publisher's website.

- The final author version and the galley proof are versions of the publication after peer review.

- The final published version features the final layout of the paper including the volume, issue and page numbers.

Link to publication

\footnotetext{
General rights

- You may freely distribute the URL identifying the publication in the public portal. follow below link for the End User Agreement:

www.tue.nl/taverne

\section{Take down policy}

If you believe that this document breaches copyright please contact us at:

openaccess@tue.nl

providing details and we will investigate your claim.
}

Copyright and moral rights for the publications made accessible in the public portal are retained by the authors and/or other copyright owners and it is a condition of accessing publications that users recognise and abide by the legal requirements associated with these rights.

- Users may download and print one copy of any publication from the public portal for the purpose of private study or research.

- You may not further distribute the material or use it for any profit-making activity or commercial gain

If the publication is distributed under the terms of Article $25 \mathrm{fa}$ of the Dutch Copyright Act, indicated by the "Taverne" license above, please 


\title{
Stochastic Theory for the Glassy State. 1. Influence of Formation History on the Glass Transition Temperature and Equation of State
}

\author{
S. Vleeshouwers and E. Nies* \\ Eindhoven University, Department of Polymer Technology, Center for Polymers and \\ Composites, P.O. Box 513, 5600 MB Eindhoven, The Netherlands
}

Received June 18, 1992; Revised Manuscript Received August 24, 1992

\begin{abstract}
A stochastic theory is developed to describe the influence of glass formation history on the equation of state of polymer glasses and on the glass transition temperature. The theory is based on the assumption of a spatial distribution of free volume and hence mobility in the polymer system. The free volume is identified with the order parameter $h$ in the Holey Huggins theory. The response of the system to a change in pressure and/or temperature is formed by a change of the actual free volume distribution to the new equilibrium. All requisite parameters but one can be obtained from independent experiments on the equilibrium polymer melt. The remaining parameter is presently determined from a nonequilibrium relaxation experiment. For poly(vinyl acetate) the calculated cooling rate and pressure dependence of the glass transition temperature and the calculated dependence of the equation of state of the polymer glass on formation pressure are compared to experimental data. In general, good agreement is obtained.
\end{abstract}

\section{Introduction}

The equilibrium equation of state behavior of polymer liquids has received considerable attention. According to equilibrium thermodynamics and statistical mechanics, the thermodynamic state is determined by a limited set of macroscopic variables. For example, the volume of a one component fluid is uniquely determined if temperature and pressure are given. Applying equilibrium statistical mechanics, it is possible to correlate this macroscopic behavior with molecular interactions. The hole theory of Simha and Somcynsky ${ }^{1}$ (SS) is considered one of the most successful equation of state theories. ${ }^{2-7}$ Recently, the "Holey Huggins" hole theory ${ }^{6}(\mathrm{HH})$, closely related to the Simha-Somcynsky theory, has been derived by one of the authors. The liquidlike structure is approximated by the introduction of a fraction of empty cells $h$ on the lattice, which at equilibrium is determined by minimization of the Helmholtz free energy. Changes in macroscopic volume reflect the corresponding microscopic changes, i.e. changes in the fraction empty cells and changes in the volume of the cell. In most cases the description obtained with the limited set of (three) molecular parameters is within the experimental accuracy of the data.

The theoretical understanding of the glassy-state behavior in general and of the equation of state of glassy polymers and the glass transition in particular is one of the central problems in condensed matter properties. Several approaches can be distinguished. Following prior work, in one of the theoretical approaches the glass transition is treated from a equilibrium thermodynamics point of view. ${ }^{8}$ The glass transition has also been assumed to be correlated with a phase transition at a temperature below the actual glass transition temperature. ${ }^{9-11}$ Another view is presented in the mode-coupling theory. According to this theory the coupling of density fluctuations reaches a critical value around the glass transition temperature, which causes a breakdown of the ergodicity of the glass. ${ }^{12-14}$ Finally, as is pursued in this work, the experimentally found glass transition is considered to be only a consequence of a gradual freeze, ${ }^{15-17}$ resulting in large molecular relaxation times in comparison with observation times.

In contrast with polymer liquids the same limited set of macroscopic variables does not suffice to fix the thermodynamic state of polymer glasses. From the experimental results of, e.g., McKinney and Goldstein, ${ }^{18}$
Greiner and Schwarzl, ${ }^{19}$ and Kogowski and Filisko ${ }^{20}$ it is clearly established that also the formation history (i.e. the route of preparation) influences the equation of state and the other properties of the polymer glass. As far as the SS hole theory is concerned, as a consequence of the nonequilibrium the evaluation of the order parameter $h$ by minimization is not applicable anymore; $h$ has to be extracted from experimental $p V T$ data from the glass and varies in the glassy state with temperature and pressure. ${ }^{21}$ Furthermore the glass transition temperature cannot be identified as an iso free volume state. ${ }^{21}$

Another aspect of the nonequilibrium condition of the polymer glass is the time dependency of physical properties, e.g. volume and enthalpy relaxation. A discussion of the kinetic aspects of these so-called physical aging phenomena is beyond the applicability of the thermodynamic theories discussed so far, and extra information concerning the dynamics of the physical changes must be introduced. A large group of theories approach these kinetics phenomenologically. ${ }^{22-24}$ Ngai et al. ${ }^{25,26}$ viewed the kinetics of relaxation processes as the result of cooperative rearrangements of small regions. Robertson ${ }^{27}$ also discussed the dynamics of relaxation separately from the description of the equilibrium state. He assumed a priori that the relaxation behavior is a reflection of the system approaching the underlying equilibrium. From a consideration of the possible rearrangements of (sub)molecular units and possible realizations in the dense disordered glassy state, Robertson arrived at a stochastic theory for the dynamics of, e.g., volume relaxation. It was argued ${ }^{27}$ that the rate of segmental rearrangements is not only determined by the average free volume but also, and even more importantly so, by the fluctuations in free volume.

In subsequent applications Robertson, Simha, and Curro (RSC) ${ }^{28}$ identified the free volume and fluctuations in free volume with the order parameter $h$ derived from the SS hole theory and the corresponding fluctuations in $h$, respectively. In general free volume is a loosely defined concept. The identification of the free volume with the structure parameter $h$ remedies this. Hence, the concepts of free volume and disorder parameter $h$ will be used interchangeably. The authors analyzed the volume relaxation behavior in poly(vinyl acetate) and polystyrene glasses after single temperature and pressure jumps and 
predicted the memory effects accompanying multiple jumps. ${ }^{28-30}$ Proceeding along these lines a direct evaluation of the influence of the formation history (e.g. cooling rate) is impossible. However, on the basis of the same stochastic theory, we would like to consider the properties of the polymer glass as a result of the formation history, retraceable to the equilibrium polymer liquid.

\section{IIa. Dynamics of Relaxation Phenomena: Stochastic Theory}

It is assumed that the relaxation behavior governing the glass transition region can be described by the approach to equilibrium of the conformational degrees of freedom of the polymer backbone. Vibrational and side group rotational degrees of freedom are assumed to be sufficiently fast and thus to respond almost instantaneously to variations in thermodynamic variables.

Consider now a change of temperature and/or pressure in the system from an initial to a final state. The thermodynamic properties change accordingly. It is our aim to monitor the kinetics of the changes. On a microscopic level this change in macroscopic properties is caused by rearrangements in the polymer chains. A discussion of the interrelation between microscopic and macroscopic changes has been given by Robertson in the context of the relaxation behavior in polymer glasses. ${ }^{27}$ The importance of the segmental rearrangements in the isolated chain and the influence of the surrounding segments in the dense disordered state have been explained. Furthermore the rate of change in the small volume element, containing the rearranging segments, is not only determined by the local segmental mobility but also by the possibility of the environment to assimilate the necessary changes.

However, our main interests are the kinetics of macroscopic properties and it is hoped that less detailed information concerning the microscopic state of the system should suffice to discuss these relaxation phenomena. It was suggested by Robertson ${ }^{27}$ that the local free volume is an appropriate parameter to describe the local segmental mobility and thus the rate of rearrangements. The system is thought to be subdivided into small volume elements, each with its (local) free volume. The sample is thus characterized by a (time-dependent) free volume distribution. For mathematical convenience it is assumed that this distribution is a set of $n$ discrete levels $\left\{w_{i}(t), i=1\right.$, $n$ :

$$
w(t)=\left\{w_{i}(t), i=1, n\right\}=\left[w_{1}(t) \cdot w_{i}(t) \cdot w_{n}(t)\right]
$$

with the local free volume in level $w_{i}$ given by $(i-1) \beta$ and $\beta$, the width of the free volume level, defined later.

Changes in the occupation of the levels occur by transitions between the different levels and can be regarded as a stochastic process known as a Markov chain, which has the important property of the Chapman-Kolmogorov condition; the transition probability $P_{i j}$ that the system changes from state $i$ to state $j$ in the time $(t+s)$ is equal to

$$
P_{i j}(t+s)=\sum_{k=1}^{n} P_{i k}(t) P_{k j}(s) \quad 1 \leq i, j, k \leq n
$$

The time derivative of this equation with respect to $s$ yields a set of coupled differential equations which can be written in matrix form

$$
\frac{\mathrm{d}}{\mathrm{d} t} P(t)=P(t) \cdot \mathbf{A}
$$

with $P(t)$ the transition probability and $\mathbf{A}$ the generator matrix, respectively, with the elements $\mathbf{A}_{i j}=\lim s \rightarrow 0$ $\mathrm{d}\left(P_{i j}(s)\right) / \mathrm{d} s$. The formal solution of eq 3 is given by

$$
P(t)=P(0) e^{\mathbf{A} t}
$$

where $P(0)$ is the initial transition probability matrix, in all applications set equal to the identity matrix $I$. In an infinitesimal time interval $(s \rightarrow 0)$, only transitions between adjacent levels can occur. Then the transition probabilities can be written in terms of transition rates

$$
\begin{gathered}
P_{i, i-1}=s \lambda_{i, i-1}+O(s) \\
P_{i, i+1}=s \lambda_{i, i+1}+O(s) \\
P_{i, i}=1-s\left(\lambda_{i, i-1}+\lambda_{i, i+1}\right)+O(s) \\
P_{i, j}=O(s) \quad \text { for } j \neq i,(i-1),(i+1)
\end{gathered}
$$

where $\lambda_{i, i-1}$ and $\lambda_{i, i+1}$ are the downward and upward transition rates from state $i$ to state $(i-1)$ and state $(i+$ 1 ), respectively, and $O$ is a quantity that approaches zero faster than $s$. Equation 4 can be solved by an eigenvalue analysis described in many textbooks. ${ }^{31}$ Once the transition probability is known, the calculation of the occupation levels is straightforward:

$$
w(t+s)=w(t) P(s)
$$

Equation 6 defines the time evolution of the occupation of the free volume levels. It can be shown that at sufficiently long times the Markov chain evolves to a stationary distribution $\left\{\xi_{i}, i=1, n\right\}$. For this stationary distribution, the up and downward transition rates are related by the balance equations

$$
\lambda_{i, i+1} \xi_{i}=\lambda_{i+1, i} \xi_{i+1} \quad 1 \leq i \leq(n-1)
$$

This stationary distribution can be identified with the equilibrium condition for the system since it has been assumed a priori that the relaxation behavior of the system is due to the approach to the equilibrium state. It is important to note that according to this theory the system will definitely reach the equilibrium state if the system is left for a sufficiently long time. Information about this equilibrium state can be derived from equilibrium thermodynamics and statistical mechanics.

The stochastic theory was applied by Robertson, Simha, and Curro to study the volume relaxation behavior of polymers subjected to single and combined temperature and pressure jumps in the glass transition region. ${ }^{28-30}$ The authors identified the free volume parameter, appearing in the stochastic model, with the order parameter $h$ defined in the SS theory. It is again emphasized that by this assumption the free volume has been defined consistently. In this contribution we will use the HH theory to extract information concerning the order parameter $h$ in the equilibrium state of the polymer system. In the following paragraph a short recapitulation of the relevant $\mathrm{HH}$ equations is given.

\section{IIb. Holey Huggins Equation of State Theory: Free Volume (Distribution)}

According to the $\mathrm{HH}$ theory ${ }^{6}$ the correlation between volume $V$, pressure $p$, and temperature $T$ for a component consisting of $N$ molecules is modeled on a lattice with lattice cells of variable volume either singly occupied or vacant. A fraction $h$ of the lattice cells is assumed to be free. The main structure parameter in this theory is the external contact fraction $q$, related to the occupied site 
fraction $y$

$$
q=(1-\alpha) y /(1-\alpha y)
$$

with $\alpha=(2 / z)(1-1 / s), y=(1-h), z$ the lattice coordination number, and $s$ the number of lattice sites occupied by a molecule of molar mass $M$. The SS and HH theories are identical for monomers $s=1$ and in the limit of infinite coordination number $z$. Furthermore at sufficiently low temperatures both theories become practically identical. However differences between SS and $\mathrm{HH}$ are important, as can be observed from, e.g., miscibility behavior. ${ }^{6,7}$

According to the $\mathrm{HH}$ theory the pressure is given by

$$
p=-(\partial A / \partial V)_{N, T, y}-(\partial A / \partial y)_{N, T, V}(\partial y / \partial V)_{N, T}
$$

At equilibrium it is assumed that the Helmholtz free energy is minimized with respect to the structure parameter $q$ or $y$ :

$$
(\partial A / \partial y)_{N, T, V}=0
$$

In reduced form the equation of state and minimization condition deduced from eqs 9 and 10 obey a practical principle of corresponding states and are defined by

$$
\begin{gathered}
\frac{\tilde{p} \tilde{V}}{T}=\frac{1}{(1-\eta)}+\frac{2(1-\alpha) y}{(1-\alpha y) \tilde{T}(y \tilde{V})^{2}}\left[\frac{A}{(y \tilde{V})^{2}}-B\right] \\
1-\frac{1}{s}+\frac{1}{y} \ln (1-y)-\frac{z}{2 y} \ln (1-\alpha y)-\frac{\alpha z}{2}= \\
c_{8}\left\{\frac{(3 \eta-1+\alpha y)}{(1-\eta)(1-\alpha y)}+\frac{(1-\alpha) y}{2 \tilde{T}(1-\alpha y)^{2}(y \tilde{V})^{2}}\left[2 B-\frac{3 A}{(y \tilde{V})^{2}}+\right.\right. \\
\left.\left.4 \alpha y\left(\frac{A}{(y \tilde{V})^{2}}-B\right)\right]\right\}
\end{gathered}
$$

with $\eta=2^{-1 / 6}(1-\alpha) y /(y \tilde{V})^{1 / 3}(1-\alpha y), \tilde{p}=p / p^{*}, \tilde{V}=V / V^{*}$, $\widetilde{T}=T / T^{*}, A=1.011, B=1.2045$, and $c_{\mathrm{s}}=$ degrees of freedom per segment.

At a given pressure $p$ and temperature $T$ the corresponding equilibrium values of the macroscopic volume $V$ and the corresponding fraction of vacant lattice cells $\langle h\rangle_{\text {eq }}$ can be calculated. According to fluctuation theory ${ }^{32}$ the fluctuation in $h$, i.e. $\delta h_{\mathrm{eq}}$, due to the thermal energy, can be computed from

$\left\langle\left(\delta h_{\mathrm{eq}}\right)^{2}\right\rangle=\left\langle\left(h-\langle h\rangle_{\mathrm{eq}}\right)^{2}\right\rangle=k T\left[\left(\partial^{2} A / \partial y^{2}\right)_{N, T, V}\right]^{-1}$

According to the HH theory, eq 13 reads, considering a volume containing $N s$ segments

$$
\begin{gathered}
\left\langle\left(\delta h_{\text {eq }}\right)^{2}\right\rangle=\frac{1}{N s y^{2}}\left\{1-\frac{1}{s}+\frac{1}{1-y}+\frac{2}{y} \ln (1-y)-\frac{\alpha z}{2}[1+\right. \\
\left.\frac{1}{(1-\alpha y)}\right]-\frac{z}{y} \ln (1-\alpha y)+c_{s}[1+ \\
\frac{\eta\left[3 \alpha y(1-\eta)+\frac{1}{3}(2+\alpha y)^{2}\right]}{(1-\alpha y)^{2}(1-\eta)^{2}}-\frac{(2+\alpha y) \eta}{(1-\alpha y)(1-\eta)}+ \\
\frac{y(1-\alpha)}{(1-\alpha y)^{3} \tilde{T}(y \tilde{\eta})^{2}}\left(\frac{\left(10 \alpha^{2} y^{2}-15 \alpha y+6\right) A}{(y \bar{\eta})^{2}}+\right. \\
\left.\left.\left.2\left(-3 \alpha^{2} y^{2}+3 \alpha y-1\right) B\right)\right]\right\}^{-1}
\end{gathered}
$$

For sufficiently small fluctuations it can be shown that the fluctuations have a Gaussian distribution with mean and variance given by $\langle h\rangle_{\text {eq }}$ and $\left\langle\left(\delta h_{\text {eq }}\right)^{2}\right)$, respectively. For numerical convenience the continuous distribution is approximated by $n$ discrete levels. It is trivial that the distribution should be zero for $h\langle 0$ and $h\rangle 1$. Keeping in mind that the normal distribution is practically zero for deviations larger than 4 times the variance, these boundary conditions will not cause any problems in the discrete representation if the continuous distribution is discretized in $n$ levels with the width of a free volume level $\beta$ :

$$
\beta=\frac{\langle h\rangle_{\mathrm{eq}}-4 \sqrt{\left\langle\left(\delta h_{\mathrm{eq}}\right)^{2}\right\rangle}}{n}
$$

According to this scheme the following free volume distribution is obtained

$$
\begin{gathered}
\xi_{i}=\Gamma\left[\beta\left(i-\frac{1}{2}\right)\right]-\Gamma\left[\beta\left(i-\frac{3}{2}\right)\right] \quad i=2, n-1 \\
\xi_{1}=\Gamma\left[\beta\left(\frac{1}{2}\right)\right] \\
\xi_{n}=1-\Gamma\left[\beta\left(n-\frac{1}{2}\right)\right]
\end{gathered}
$$

with $\Gamma(x)$ the cumulative normal distribution ${ }^{33}$ with mean $\langle h\rangle_{\text {eq }}$, variance $\left\langle\left(\delta h_{\text {eq }}\right)^{2}\right\rangle$, evaluated at $x$.

\section{IIc. Transition Rates: Kinetics}

In order to use the stochastic theory the upward and downward transition rates $\lambda_{i, i+1}$ and $\lambda_{i+1, i}, 1 \leq i \leq(n-1)$, respectively, must be defined. It was argued ${ }^{27}$ that the rate of change in a small-volume element is not only determined by the local segmental mobility but also by the possibility for the neighboring volume elements to assimilate these changes. Although, at equilibrium, correlations in the free volume content between neighboring volume elements are absent, this is not true away from equilibrium. However, it is assumed that the correlations remain small and that the free volume of the neighbors can be set equal to the average free volume $\langle h\rangle$ of the system. Hence the transition rates in a central element out of state $i$ depend on the regional free volume $h_{i}$

$$
h_{i}=\frac{(i-1) \beta+\left(z_{n}-1\right)\langle h\rangle}{z_{n}} \quad i=1, n
$$

where $z_{n}$ is the number of regions necessary to liberate or absorb the free volume changes in the central region.

Finally, the functional dependence of the local transition rates on regional free volume $h_{i}$ is assumed to be identical with the dependence of the global mobility $\mu$ on the equilibrium overall free volume $\langle h\rangle_{\text {eq. }}$. The global mobility of a system with free volume $h$ can be expressed, e.g., by a Williams-Landel-Ferry (WLF) type of equation. On a local level this reads

$$
\mu_{\mathrm{i}} \propto \exp \left(2.303 \frac{c_{1}^{\prime}\left(h_{i}-f_{\mathrm{o}}^{\prime}\right)}{c_{2}^{\prime}+h_{i}-f_{\mathrm{o}}^{\prime}}\right)
$$

For the local transition rates the following expressions are used

$$
\begin{aligned}
& \lambda_{i, i-1}=\frac{R}{\beta^{2}} \mu_{i}\left(\xi_{i-1} / \xi_{i}\right)^{1 / 2} \\
& \lambda_{i, i+1}=\frac{R}{\beta^{2}} \mu_{i}\left(\xi_{i+1} / \xi_{i}\right)^{1 / 2}
\end{aligned}
$$

where $R\left(\mathrm{~s}^{-1}\right)$ contains a characteristic rate and a com- 
pensation for differences in global and local mobilities and the occurrence of $\xi_{i}$ 's assures detailed balancing (eq 7). The factor $\beta^{-2}$ arises from the random walk representation.

\section{Stochastic Simulation}

We are now in a position to study the dynamics of free volume relaxation. The following procedure is followed.

\section{IIIa. Simulation Procedure}

1. At a given $T$ and $p$, well in the equilibrium liquid state, the corresponding $V,\langle h\rangle_{\mathrm{eq}}$, and $\left\langle\left(\delta h_{\mathrm{eq}}\right)^{2}\right\rangle$ are computed (eqs 11,12, and 14).

2. These thermodynamic data are used to specify the complete Gaussian distribution $\left\{\xi_{i}, i=1, n\right\}$ (eqs 15 and $16)$.

3. The actual distribution $\left\{w_{i}(t), i=1, n\right\}$ is set equal to the equilibrium distribution.

After preparation of the initial distribution, the actual simulation of, e.g., a cooling, a pressurizing, or a combined experiment is started. The numerical simulation procedure will be illustrated for a cooling experiment. A polymer melt which is initially, at time $t$, at temperature $T$ and pressure $p$, is cooled at a rate $T^{\prime}(\mathrm{K} / \mathrm{s})$. At a certain temperature the glass transition temperature will be reached, and upon further cooling, a polymer glass is formed.

The numerical simulation of this real time experiment proceeds along the following scheme. The specified cooling rate assures the existence of a one to one correspondence between time and temperature. According to the following procedure the stochastic process is performed.

1. Calculate the equilibrium properties $\left(V,\langle h\rangle_{\text {eq }}\right.$, $\left.\left\langle\left(\delta h_{\mathrm{eq}}\right)^{2}\right\rangle\right)$ at $T$ and $p$ corresponding to a time $t+\Delta t$.

2. Calculate the equilibrium distribution $\left\{\xi_{i}, i=1, n\right\}$ at $t+\Delta t$.

3. Compute the transition rates using the equilibrium distribution and the actual distribution $\left\{w_{i}(t), i=1, n\right\}$ at time $t$.

4. Determine the transition probabilities $P(t)$ (eq 4).

5. Update the actual distribution $\left\{w_{i}(t+\Delta t), i=1, n\right\}$ (eq 6) at time $t+\Delta t$.

6. Increment the time with $\Delta t$, and repeat steps 1-6.

The time step $\Delta t$ is chosen sufficiently small to assure that the equilibrium free volume changes only approximately $5 \times 10^{-4}$. Typically, this corresponds to a temperature change of $1 \mathrm{~K}$ or a pressure change of $2 \mathrm{MPa}$. The matrix A (eq 4) will not change significantly during $\Delta t$, and the process between $t$ and $t+\Delta t$ can be treated as a Markov chain.

A similar experiment can be done by, e.g., specifying a pressurizing rate $p^{\prime}$ or by a simultaneous change in $T$ and $p$. Furthermore $T^{\prime}$ and $p^{\prime}$ can be changed at will during the simulation. For example, at a given $T$ and $p$ in the glassy state one can set $T^{\prime}=0$ and $p^{\prime}=0$ and from that time on monitor the physical aging. It is thus possible to simulate the complete formation history of a polymer glass and study the influence of these parameters on the resulting thermal properties.

\section{IIIb. General Results Obtained in a Simulation Run}

During the simulation and actual distribution $w(t)$ and the calculated average free volume $\langle h(t)\rangle_{\mathrm{ac}}$ can differ from the equilibrium values. To depict the simulation results the actual average free volume value can be shown (see, e.g., Figure 5).
Table I

Parameters for PVAC, Used in Simulations

\begin{tabular}{llll}
\hline$p^{*}(\mathrm{MPa})$ & 895.5 & $f_{o}^{\prime}$ & $0.11\left(0.086^{a}\right)$ \\
$V^{*}\left(\mathrm{~cm}^{3} / \mathrm{g}\right)$ & 0.8063 & $R\left(\mathrm{~s}^{-1}\right)$ & $1\left(10^{-6 a}\right)$ \\
$T^{*}(\mathrm{~K})$ & 7988 & $N_{\mathrm{s}}$ & 26 \\
$c_{1}^{\prime}$ & $9\left(12.8^{a}\right)$ & $z_{\mathrm{n}}$ & 13 \\
$c_{2}{ }^{\prime}$ & $0.061\left(0.017^{a}\right)$ & $n$ & 20
\end{tabular}

"WLF parameters derived by Plazek from data in the "softening" region. ${ }^{38}$

At sufficiently high temperatures the actual and equilibrium values (and of course also the complete distributions) coincide; thus the system is equilibrated. At a certain temperature the actual distribution $w(t)$ and also the actual average free volume $\langle h(t)\rangle_{\text {ac }}$ commence to deviate from the equilibrium condition. Upon further cooling, the difference between actual and equilibrium states increases. As depicted in, e.g., Figure 4 a transition temperature $T_{\mathrm{g}}$ can be obtained as the intercept of the extrapolated "glassy" part of the curve with the equilibrium line.

Furthermore the $p, h, T$ space obtained from a specific simulation experiment can be used to compute the thermodynamic properties. As an illustration, consider the computation of the volume $V$. In the equilibrium state (temperatures higher than the transition temperature $T_{\mathrm{g}}$ ) the equation of state is given by eq 11. For temperatures and pressures in the nonequilibrium part of the diagram, eq 11 is in principle not valid. In this case the equation of state must be obtained from the full pressure equation (eq 9). In earlier work Simha and co-workers ${ }^{21}$ have compared the calculation of the volume $V$ according to the full pressure equation (eq 9 ) with the calculation based on only eq 11. It was shown that for the $p V T$ behavior the differences between the two approaches are small. In the present contribution it has been confirmed that the differences in volume $\Delta V$ obtained from the full equation (eq 9) or the minimized equation of state (eq 11) are only minor $\left(\Delta V<10^{-5} \mathrm{~cm}^{3} / \mathrm{g}\right)$. Consequently, the results reported here are obtained from eq 11.

\section{Results and Discussion}

The stochastic theory will be applied to the thermal behavior of poly(vinyl acetate) (PVAC), because for this polymer experimental volume relaxation data are available.

\section{IVa. Determination of the Different Parameters}

The thermodynamic scaling parameters $p^{*}, V^{*}$, and $T^{*}$, estimated from experimental $p V T$ data in the liquid state determined by McKinney and Goldstein, ${ }^{18}$ are summarized in Table I. The details concerning the estimation procedure and the multiparameter estimation program used to obtain the parameters are described elsewhere. ${ }^{34}$ The description of the liquid $p V T$ data is within the accuracy of the experimental data, which is $\Delta V= \pm 5 \times 10^{-4} \mathrm{~cm}^{3} / \mathrm{g}$. With the scaling parameters, $\langle h\rangle_{e q}$ and also the fluctuations $\left\langle\left(\delta h_{\mathrm{eq}}\right)^{2}\right\rangle$ can be calculated from eqs 11 and 12 and eq 14 , respectively.

Subsequently, the full $h$ distribution $\left\{\xi_{i}, i=1, n\right\}$ for $n$ $=20$ is calculated, using eqs 15 and 16 . For this number of states an accurate discrete representation of the continuous distribution and still reasonable computation times are obtained.

The parameters $c^{\prime}{ }_{1}, c^{\prime}{ }_{2}$, and $f^{\prime}{ }_{\circ}$ describing the correlation between global mobility and free volume are obtained from experimental viscosimetric shift factors. The data, measured at ambient pressure as a function of temperature in the liquid state, are taken from Williams and Ferry. ${ }^{35}$ The free volume corresponding to the experimental pressure 


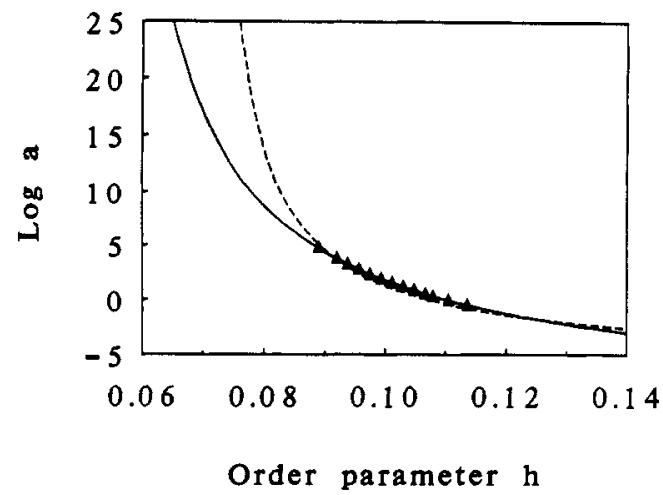

Figure 1. Experimental dynamic mechanical shift factors versus order parameter $h(\Delta)$ for PVAC. WLF fit (lines): (solid line) best fit; (dotted line) parameters derived from Plazek. ${ }^{38}$

and temperatures are calculated from eqs 11 and 12 by adapting the scaling parameters shown in Table I. The parameters $c_{1}^{\prime}, c^{\prime}{ }_{2}$, and $f_{0}^{\prime}$ are estimated using the same multiparameter estimation program mentioned earlier. For our present purposes, extrapolation of the function to low $h$ values is necessary. The description of the shift factors $a=1 / \mu_{\langle h\rangle}$ by eq 18 is shown in Figure 1 by the full line.

The only parameter that must be obtained from dynamic information is the constant $R$ in eqs 19 . It will become clear that this parameter can be estimated from different kinds of experiments. In this first application we use the relaxation in a jump experiment (Kovacs ${ }^{36}$ ) to obtain a value for $R$, as is also done by RSC. 28 A change in the value of $R$ shifts the relaxation pattern horizontally, i.e. along the time axis, without changing the shape of the relaxation curve. The best description of these experiments (shown in Figure 2) yields the value for the constant $R$ given in Table I. It should be noted that the numerical value of $R$ has no real physical significance but is merely an adjustable parameter. In this and all subsequent simulations also the parameters $N_{\mathrm{s}}$ and $z_{n}$ are fixed to literature values. ${ }^{28}$ Changes due to the choice of different numerical values for $N_{\mathrm{s}}$ and/or $z_{n}$ can be compensated by a change in the effective $R$ value. The overall shape of the relaxation curve only changes slightly. The complete set of parameters, summarized in Table $I$, is now fixed, and we are ready to explore other dynamic simulations.

\section{IVb. Simulation Results}

In Figure 2 the computed volume relaxation behavior brought about by single temperature jumps is compared to experimental results. The observed agreement is typical for the present implementation of the stochastic theory as observed earlier by RSC. ${ }^{28}$ Subsequently, they showed that the agreement can be improved by adopting a temperature-dependent $R$ and more refined functions correlating the macroscopic mobility and free volume. ${ }^{20-30,37}$ For the present purpose we will accept the observed deviations, bearing in mind how they can be resolved, and proceed with other simulation results.

In Figure 3 and Table II the simulated glass transition temperatures at ambient pressure are presented as a function of cooling rate. In the simulations the glass transition temperature is identified with the intercept of extrapolated glass and the liquidlike volume traces. The simulated cooling rate dependence of $T_{\mathrm{g}}$ is not linear. In Table II the average value is listed. An important observation is that the best $R$ value derived from the jump experiment gives an accurate prediction of the glass transition temperature $T_{\mathrm{g}}$ at atmospheric pressure. This
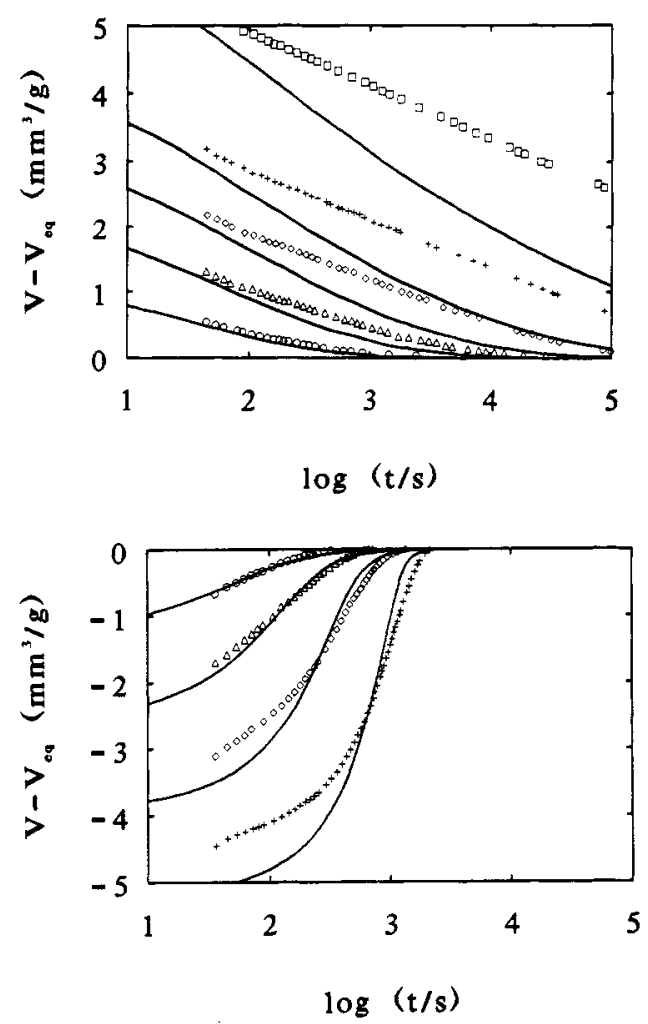

Figure 2. Volume departure from equilibrium versus time after single temperature jumps for PVAC. Symbols, experimental data (Kovacs ${ }^{36}$ ): (top) jumps from 40 to $37.5^{\circ} \mathrm{C}(0), 35^{\circ} \mathrm{C}(\Delta), 32.5^{\circ}$ $\mathrm{C}(\diamond), 30^{\circ} \mathrm{C}(+)$, and $25^{\circ} \mathrm{C}(\mathrm{C})$; (bottom) jumps to $40^{\circ} \mathrm{C}$ from $37.5^{\circ} \mathrm{C}(0), 35^{\circ} \mathrm{C}(\Delta), 32.5^{\circ} \mathrm{C}(\diamond)$, and $30^{\circ} \mathrm{C}(+)$. Solid lines, calculated.

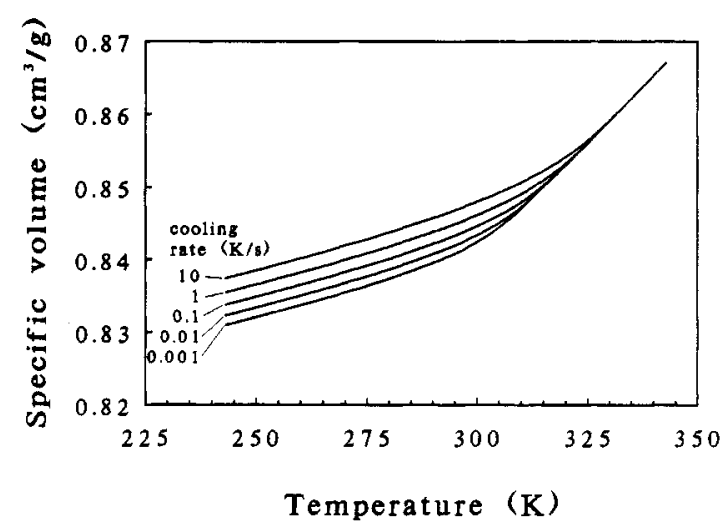

Figure 3. Calculated volume versus temperature for indicated cooling rates for PVAC.

suggests that also the volumetric glass transition temperature, which is more commonly available than volume relaxation experiments, can be used to estimate the parameter $R$, which enables prediction of the volume relaxation behavior for a given polymer under different conditions.

The influence of pressure on the formation of the glassy state is shown in Figure 4. The simulated formation conditions are choosen to mimic the experimental ${ }^{18}$ formation history ( $p$ and cooling $T^{\prime}$ ) (see Table II). The dependence of volume on temperature under isobaric cooling is depicted. The agreement for the $0.1-\mathrm{MPa}$ curve is excellent: maximum deviations between experimental and theoretical specific volumes $\Delta V$ do not exceed $2 \times$ $10^{-3} \mathrm{~cm}^{3} / \mathrm{g}$. At higher pressures the predicted specific volumes are systematically too large but the maximum deviation $\Delta V$ remains less than $8 \times 10^{-3} \mathrm{~cm}^{3} / \mathrm{g}$ at $p=80$ $\mathrm{MPa}$. Values for the thermal expansion coefficient $\alpha_{\mathrm{g}}$ and the glass transition temperature $T_{\mathrm{g}}$ extracted from the 
Table II

Comparison of Calculated Quantities ${ }^{39}$ with Experimental Data ${ }^{18}$ for PVAC

\begin{tabular}{|c|c|c|c|c|c|}
\hline \multirow[b]{2}{*}{ property } & \multirow[b]{2}{*}{$\exp$} & \multirow[b]{2}{*}{ calc } & \multicolumn{3}{|c|}{ formation history } \\
\hline & & & $p(\mathrm{MPa})$ & cooling rate $(\mathrm{K} / \mathrm{s})$ & figure \\
\hline \multirow{5}{*}{$T_{\mathrm{g}}(\mathrm{K})$} & & 314.5 & 0.1 & 10 & 3 \\
\hline & & 310.0 & 0.1 & 1 & 3 \\
\hline & & 306.2 & 0.1 & 0.1 & 3 \\
\hline & & 303.1 & 0.1 & 0.01 & 3 \\
\hline & & 300.7 & 0.1 & 0.001 & 3 \\
\hline \multirow{6}{*}{$\begin{array}{l}\mathrm{d} T_{\mathrm{g}} / \mathrm{d}(\log \text { cooling rate })(\mathrm{K}) \\
T_{\mathrm{g}}(\mathrm{K})\end{array}$} & & 3.5 & 0.1 & $0.001-10$ & 3 \\
\hline & 304.7 & 301.0 & 0.1 & 0.00139 & 4,6 \\
\hline & 309.7 & 307.3 & 20 & 0.00139 & 4 \\
\hline & 314.2 & 314.0 & 40 & 0.00139 & 4 \\
\hline & 321.6 & 327.1 & 80 & 0.00139 & 4 \\
\hline & 304.7 & $303.0^{a}$ & 0.1 & 0.00139 & \\
\hline \multirow{3}{*}{$\begin{array}{l}\mathrm{d} T_{\mathrm{g}} / \mathrm{d} p\left(\mathrm{~K} \mathrm{MPa}^{-1}\right) \\
\alpha(0.1 \mathrm{MPa} \text { glass, } 273 \mathrm{~K})\left(\mathrm{K}^{-1}\right)\end{array}$} & 0.212 & 0.33 & $0.1-80$ & 0.00139 & 4 \\
\hline & $2.86 \times 10^{-4}$ & $2.24 \times 10^{-4}$ & 0.1 & 0.00139 & 4,6 \\
\hline & $2.59 \times 10^{-4}$ & $2.03 \times 10^{-4}$ & 80 & 0.00139 & 6 \\
\hline \multirow[t]{2}{*}{$\beta(0.1 \mathrm{MPa}$ glass, $273 \mathrm{~K})\left(\mathrm{MPa}^{-1}\right)$} & $2.75 \times 10^{-4}$ & $2.64 \times 10^{-4}$ & 0.1 & 0.00139 & 6 \\
\hline & $2.54 \times 101-4$ & $2.32 \times 10^{-4}$ & 80 & 0.00139 & 6 \\
\hline \multirow[t]{2}{*}{$\alpha(80 \mathrm{MPa}$ glass, $273 \mathrm{~K})\left(\mathrm{K}^{-1}\right)$} & $2.94 \times 10^{-4}$ & $1.96 \times 10^{-4}$ & 0.1 & 0.00139 & 7 \\
\hline & $2.63 \times 10^{-4}$ & $1.74 \times 10^{-4}$ & 80 & 0.00139 & 7 \\
\hline \multirow[t]{2}{*}{$\beta(80 \mathrm{MPa}$ glass, $273 \mathrm{~K})\left(\mathrm{MPa}^{-1}\right)$} & $2.70 \times 10^{-4}$ & $2.65 \times 10^{-4}$ & 0.1 & 0.00139 & 7 \\
\hline & $2.42 \times 10^{-4}$ & $2.31 \times 10^{-4}$ & 80 & 0.00139 & 7 \\
\hline \multirow{2}{*}{$\alpha(0.1 \mathrm{MPa}$ glass, $273 \mathrm{~K})\left(\mathrm{K}^{-1}\right)$} & $2.86 \times 10^{-4}$ & $1.95 \times 10^{-4 a}$ & 0.1 & 0.00139 & \\
\hline & $2.59 \times 10^{-4}$ & $1.76 \times 10^{-4} a$ & 80 & 0.00139 & \\
\hline \multirow{2}{*}{$\beta(0.1 \mathrm{MPa}$ glass, $273 \mathrm{~K})\left(\mathrm{MPa}^{-1}\right)$} & $2.75 \times 10^{-4}$ & $2.66 \times 10^{-4} a$ & 0.1 & 0.00139 & \\
\hline & $2.54 \times 10^{-4}$ & $2.30 \times 10^{-4 a}$ & 80 & 0.00139 & \\
\hline
\end{tabular}

${ }^{a}$ Calculations with WLF constants from Plazek. ${ }^{38}$

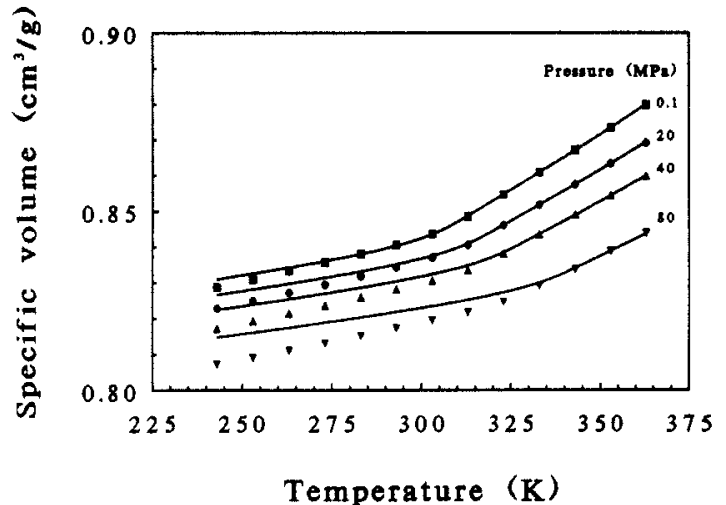

Figure 4. Volume versus temperature for indicated formation pressures for PVAC: (solid lines) calculated isobars; (symbols) experimental. ${ }^{18}$

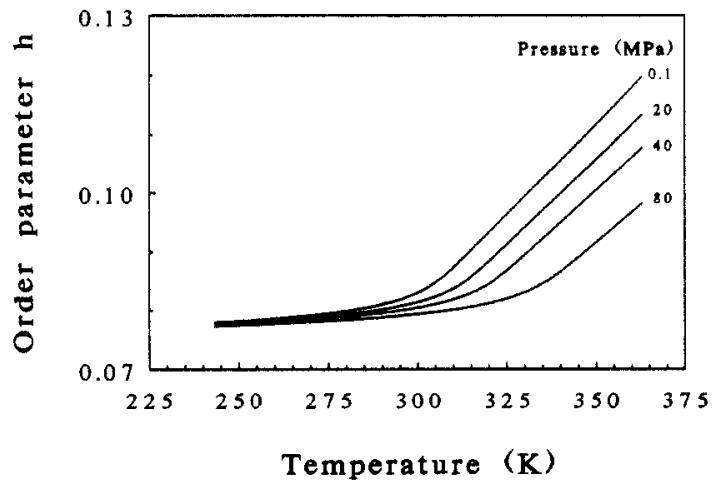

Figure 5. Order parameter $h$ versus temperature for indicated pressures for PVAC: (solid lines) calculated isobars.

simulation results are summarized and compared with experimental data in Table II. The increasing deviations with pressure between theory and experiment in the glassy state can be attributed to too small a predicted value of the pressure dependence of the glass transition temperature $\left(\mathrm{d} T_{\mathrm{g}} / \mathrm{d} p\right)$ (see Table II).

In Figure 5 the $h, T, p$ results corresponding to the $V, T, p$ data from Figure 4 are shown. From this it can be concluded that the change in specific volume in the glassy state is mainly due to changes in lattice cell volume and not so much by changes in free volume. This is in contrast with results obtained by Simha following a different approach. Simha ${ }^{21}$ extracted the free volume parameter $h$ from the experimental $p V T$ data, thus obtaining a parameter $h$ that depends on temperature and pressure.

Now it is found that the equilibrium free volume at the glass transition temperature, $h_{\mathrm{g}}$, is practically constant. Thus the glass transition can be regarded as a practical iso free volume transition. Because the value of $h_{\mathrm{g}}$ depends on cooling rate it is certainly not a material constant. The iso free volume condition seems to be related to the assumption that the mobility depends on the free volume only, eq 18. However, the predicted pressure-independent value for $h_{\mathrm{g}}$ is not in agreement with experimental data. The experimental pressure dependence of $T_{\mathrm{g}}{ }^{18}$ yields a lineair pressure dependence for $h_{\mathrm{g}}$

$$
h_{\mathrm{g}}=0.084-8.57 \times 10^{-5} p \quad p \text { in } \mathrm{MPa}
$$

The simulated $p V T$ behavior of a polymer glass formed by cooling at $0.1 \mathrm{MPa}$ and by subsequently pressurizing is shown in Figure 6 together with experimental data.18 Once more the experimental formation history was reproduced accurately (see Table II). In this case the maximum deviation between experimental and theoretical volume $\Delta V$ is smaller than $3 \times 10^{-3} \mathrm{~cm}^{3} / \mathrm{g}$. For a polymer glass formed by cooling a high pressure polymer melt ( $p$ $=80 \mathrm{MPa}$ ) and by subsequently depressurizing, the results are depicted in Figure 7, together with experimental data. ${ }^{18}$ In this case the deviations $\Delta V$ are larger $\left(\Delta V<8 \times 10^{-3}\right.$ $\mathrm{cm}^{3} / \mathrm{g}$ ) and can be mainly attributed to the overestimated pressure dependence of the glass transition temperature, as explained earlier. Finally, the estimated isothermal compressibilities $\beta_{\mathrm{g}}$ of polymer glasses formed at high and low pressure are compared with experimental data in Table II.

Figure 1 shows the experimental dynamic mechanical shift factors, used to obtain a relation between free volume and mobility. Equilibrium shift factors at small free volume content, corresponding to temperatures below $T_{\mathrm{g}}$, 


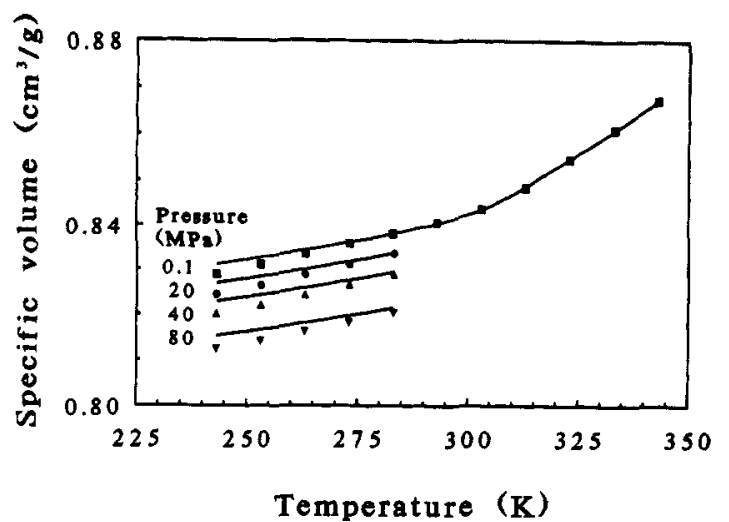

Figure 6. Volume versus temperature for a PVAC glass formed by cooling at $0.1 \mathrm{MPa}$ and subsequently pressurizing: (solid lines) calculated isobars; (symbols) experimental. ${ }^{18}$

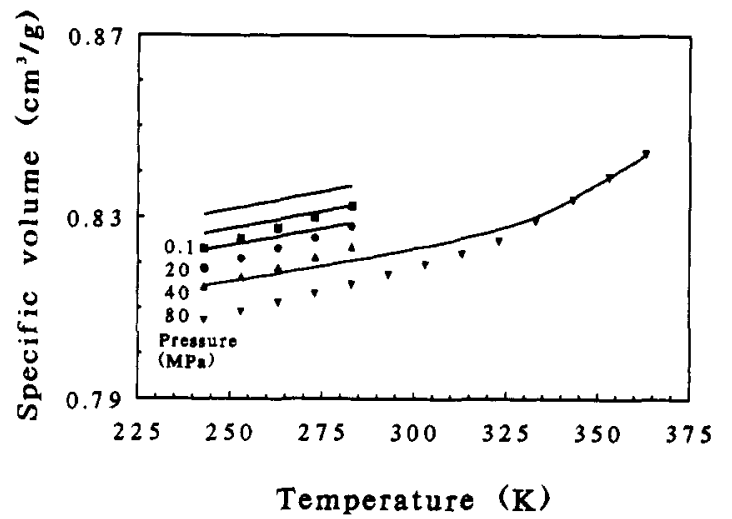

Figure 7. Volume versus temperature for a PVAC glass formed by cooling at $80 \mathrm{MPa}$ and subsequently depressurizing: (solid lines) calculated isobars; (symbols) experimental..$^{18}$

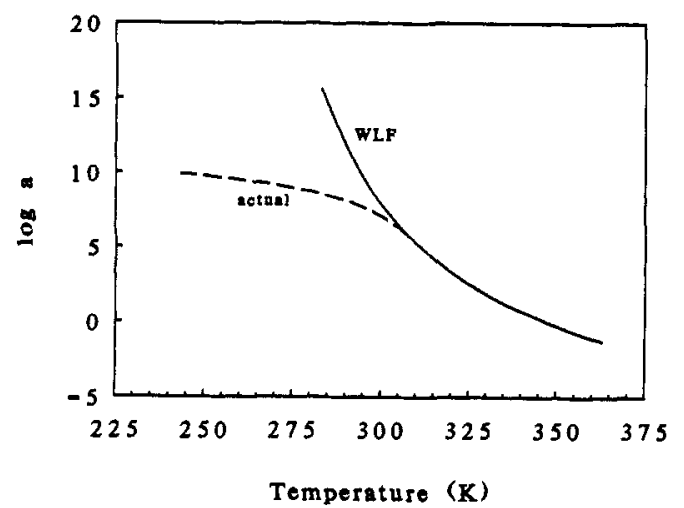

Figure 8. Shift factors of the simulated PVAC glass formed by cooling at $0.1 \mathrm{MPa}$ and subsequently pressurizing, calculated from $h$ (dotted line) or WLF (solid line).

are obtained by extrapolation. From the free volume data shown in Figure 5, the shift factors $a$ in a glass formed at atmospheric pressure can be estimated, using eq 18, with $a=1 / \mu_{\langle h\rangle}$. Results of this calculated shift factor are shown in Figure 8. As expected, the shift factors in the glass do not follow the WLF behavior, but level off at low temperatures.

So far the WLF equation depicted by the full curve in Figure 1 was used. The precise extrapolation to low $h$ values seems somewhat arbitrary and depends on (the constants of) the adapted function (WLF in our case). To examine the effect of the relation between free volume and mobility, the same simulations were performed using the dotted line in Figure 1. This line, also used by RSC, 30,37 corresponds to the WLF parameters summarized in Table I, which are derived from Plazek's data, ${ }^{38}$ obtained at temperatures $T, 308 \mathrm{~K} \leq T \leq 333 \mathrm{~K}$. With these parameters and $R=10^{-6} \mathrm{~s}^{-1}$ a best fit of the volume relaxation following a temperature jump is obtained. The faster decrease of mobility at low free volumes inferred from Plazek's curve results in volumes up to $2 \times 10^{-3} \mathrm{~cm}^{3} / \mathrm{g}$ larger and a glass transition temperature $T_{\mathrm{g}} 2 \mathrm{~K}$ higher. Furthermore, the thermal expansion coefficient $\alpha_{g}$ at 0.1 $\mathrm{MPa}$ and $273 \mathrm{~K}$ is $10 \%$ smaller compared with the value obtained from the simulation shown in Figure 6 (see Table II).

\section{Conclusions}

From equilibrium $p V T$ data, equilibrium dynamic mechanical shift factors, and only one nonequilibrium volume relaxation experiment, it is possible to predict the equation of state behavior of glasses, the glass transition temperature and its dependence on formation history (important conditions are cooling rate, pressure, pressurizing rate, temperature, and annealing time). Simulations at ambient pressure show excellent agreement with experimental data (Table II).

Discrepancies between theory and experiment at high pressures are almost entirely related to the dependence of $T_{\mathrm{g}}$ on the glass formation pressure. The use of shift factor data, obtained at ambient pressure, to obtain a relation between mobility and free volume, is probably limited to low pressures. Discrepancies at high pressures, as shown in this contribution, suggest that mobility at high pressure is not only described by the order parameter $h$, which is here used as a measure for free volume. In future work we will incorporate pressure-dependent shift factors to obtain a new relation to describe mobility.

The choice of the relation between free volume and mobility and in particular the extrapolation of this relation to low free volume influences simulation results (see Figure 1 and Table II). Fortunately, this uncertainty only causes minor differences. Comparison of simulated shift factors with experimental nonequilibrium shift factors can further clarify this relation. This comparison will be presented in a future contribution.

The only nonequilibrium data used for the predictions are volume data of a single relaxation experiment, to estimate a value for $R$ (eqs 19). Decreasing the value of $R$ by 1 order of magnitude induces an increase of $T_{\mathrm{g}}$ by $3 \mathrm{~K}$ and an increase in the volume of the glass formed at an atmospheric pressure of $\mathrm{ca} .10^{-3} \mathrm{~cm}^{3} / \mathrm{g}$. In the relaxation curves in up and down step experiments, as shown in Figure 2 , the same change in $R$ brings about a 1 decade shift to longer times. As is shown in this contribution the parameter $R$ can also be estimated from different experiments, e.g. the location of the ambient glass transition temperature.

In the near future we will apply the presented scheme to the simulation of other polymers, such as polystyrene and polycarbonate, to investigate the influence of pressure on mobility, and to the simulation of other experiments, e.g. dynamic bulk compressibility and enthalpy relexation.

Acknowledgment. Partial support of this research was received from the NATO Collaborative Research Grant, CRG 900260. SV also wishes to acknowledge support from the "Foundation Polymer Blends" SPB.

\section{References and Notes}

(1) Simha, R.; Somcynsky, T. Macromolecules 1969, $2,341$.

(2) Jain, R. K.; Simha, R.; Zoller, P. J. Polym. Sci., Polym. Phys. Ed. 1982, 20, 1399 .

(3) Simha, R.; Jain, R. K. Colloid Polym. Sci. 1985, 263, 905.

(4) Simha, R.; Nies, E.; Stroeks, A. Polym. Prepr. (Am. Chem. Soc., Div. Polym. Chem.) 1991, 32, 489. 
(5) Stroeks, A.; Nies, E.; Simha, R. Polym. Prepr. (Am.Chem. Soc., Div. Polym. Chem.) 1991, 32, 491.

(6) Nies, E.; Stroeks, A. Macromolecules 1990, 23, 4088.

(7) Stroeks, A.; Nies, E. Macromolecules 1990, 23, 4092.

(8) Breuer, H.; Rehage, G. Kolloid-Z. Z. Polym. 1967, 216-217, 159.

(9) Gibbs, J. H.; DiMarzio, E. A. J. Chem. Phys. 1958, $28,373$.

(10) Adams, G.; Gibbs, J. H. J. Chem. Phys. 1965, 43, 139.

(11) Cohen, M. H.; Grest, G. S. Phys. Rev. B 1979, 20, 1077.

(12) Leutheusser, E. Phys. Rev. B 1984, 29, 2765.

(13) Bengtzelius, U. Phys. Rev. A 1986, 33, 3433.

(14) Jäckle, J. J. Phys.: Condens. Matter 1989, 1, 267.

(15) Jäckle, J. Rep. Prog. Phys. 1986, 49, 171.

(16) Kirkpatrick, T. R.; Thiramalai, D.; Wolynes, P. G. Phys. Rev. A 1989, 40, 1045 .

(17) Donth, E. J. Non-Cryst. Solids 1991, 131-133, 204.

(18) McKinney, J. E.; Goldstein, M. J. Res. Natl. Bur. Stand.-A. Phys. Chem. 1974, 78A, 331.

(19) Greiner, R.; Schwarzl, F. R. Rheol. Acta 1984, 23, 378.

(20) Kogowski, G. J.; Filisko, F. E. Macromolecules 1986, 19, 828.

(21) McKinney, J. E.; Simha, R. Macromolecules 1976, 9, 431.

(22) Moynihan, C. T.; Eastreal, A. J.; DeBolt, M. A.; Tucker, J. J. Am. Ceram. Soc. 1976, 59, 12.

(23) Kovacs, A. J.; Aklonis, J. J.; Hutchinson, J. M.; Ramos, A. R. J. Polym. Sci., Polym. Phys. Ed. 1979, 17, 1097.

(24) Matsuoka, S.; Williams, G.; Johnson, G. E.; Anderson, E. W.; Furukawa, T. Macromolecules 1985, 18, 2652.

(25) Ngai, K. L.; Rendell, R. W.; Rajagopal, A. K.; Teitler, S. Ann. N.Y. Acad. Sci. 1988, 484, 150.
(26) Ngai, K. L.; Rajagopal, A. K.; Teitler, S. J. Chem. Phys. 1988, $88,5086$.

(27) Robertson, R. E. J. Polym. Sci., Polym. Phys. Ed. 1979, 17, 597.

(28) Robertson, R. E.; Simha, R.; Curro, J. G. Macromolecules 1984, 17,911 .

(29) Robertson, R. E.; Simha, R.; Curro, J. G. Macromolecules 1985, $18,2239$.

(30) Robertson, R. E.; Simha, R.; Curro, J. G. Macromolecules 1988, $21,3216$.

(31) Goodman, R. Introduction of Stochastic Models; Benjamin/Cummings: Menlo Park, CA, 1988; $p 127$.

(32) Landau, L. D.; Lifshitz, E. M. Course of Theoretical Physics; Pergamon Press: Oxford, U.K., 1958.

(33) Abramowitz, M.; Stegun, I. A. Handbook of Mathematical Functions; Dover Publications: New York, 1965.

(34) Hillegers, L. T. M. E. The Estimation of Parameters in Functional Relationship Models. Dissertation, Eindhoven University of Technology, The Netherlands, 1986.

(35) Williams, M. L.; Ferry, J. D. J. Colloid Sci. 1954, 9, 479.

(36) Kovacs, A. J. Fortschr. Hochpolym.-Forsch. 1963, 3, 394.

(37) Robertson, R. E. Macromolecules 1985, 18, 953.

(38) Plazek, D. J. Polym. J. (Tokyo) 1980, 12, 43.

(39) Due to the use of slightly different parameters some numerical values differ from those presented earlier: Vleeshouwers, S.; Nies, E. Polym. Commun. 1991, 32, 418.

Registry No. PVAC, 9003-20-7. 